\title{
Plantas medicinais: conhecimento e uso por usuários de Unidades Básicas de Saúde em Araruna - PB, Brasil
}

Medicinal plants: knowledge and use by users of Basic Health Units in Araruna - PB, Brazil

Plantas medicinales: conocimiento y uso por usuarios de Unidades Básicas de Salud en Araruna - PB, Brasil

Sabrina Avelar de Macêdo FERREIRA ${ }^{1}$

Diego ROMÁRIO-SILVA ${ }^{2}$

Pierre Andrade Pereira OLIVEIRA ${ }^{\mathbf{1}}$

Pedro Henrique SETTE-DE-SOUZA ${ }^{3}$

Ana Caroline RODRIGUES ${ }^{4}$

Andréa Cristina Barbosa da SILVA ${ }^{5}$

${ }^{1}$ Departamento de Odontologia, Universidade Estadual da Paraíba (UEPB), Campus VIII, 58233-000, Araruna, PB, Brasil

${ }^{2}$ Faculdade de Tecnologia do Ipê - FAIPE - 78043-172, Cuiabá, MT, Brasil

${ }^{3}$ Departamento de Odontologia, Universidade de Pernambuco (UPE), 56512-200 Arcoverde - PE, Brasil

${ }^{4}$ Departamento de Odontologia. Universidade Federal de Campina Grande, 58708-110 Patos - PB, Brasil

${ }^{5}$ Departamento de Farmácia, Universidade Estadual da Paraíba (UEPB), Campus I, 58429-500 Campina Grande, PB, Brasil

\section{Resumo}

Estudos etnobotânicos possibilitam o resgate e a preservação dos conhecimentos populares das comunidades, além do que essas informações podem ser utilizadas em pesquisas para produção de fitoterápicos no Brasil, que é ainda incipiente, apesar da sua enorme biodiversidade vegetal. Objetivamos realizar um estudo etnobotânico de plantas medicinais indicadas e utilizadas pela população de Araruna. Dois questionários estruturados diferenciados foram utilizados para a obtenção dos dados socioeconômicos e etnobotânicos, um para os raizeiros e outro para os usuários do Sistema Único de Saúde (SUS). A maioria dos entrevistados (66,5\%) faz uso de plantas medicinais. Destes, $52,63 \%$ residem na zona rural e $47,37 \%$ na zona urbana. Além disso, 27,5\% obtinham as plantas no próprio domicílio e $27 \%$ em outros locais, como na casa de parentes e amigos ou no sítio de vizinhos. Em relação a como foi adquirido o conhecimento sobre a utilização das plantas medicinais, $61 \%$ afirmaram ter obtido com a família. As plantas para fins odontológicos mais vendidas pelos raizeiros foram: boldo, camomila, canela, cajueiroroxo, alecrim, alho, babatenon, aroeira, sabugueira, cravo, romã e erva-doce. As plantas mais indicadas e utilizadas pelos usuários do Sistema Único de Saúde (SUS) foram: erva-cidreira, capim santo, boldo, sabugueira, hortelã da folha miúda, malva rosa, romã, hortelã da folha grossa, eucalipto, erva-doce, mastruz, louro, cajueiro roxo, alecrim, arruda e anador. A população de Araruna faz uso de plantas medicinais para o tratamento de diversos agravos à saúde, com conhecimento adquirido de forma popular e sem orientação profissional sobre posologia e/ou possível toxicidade dessas plantas.

Descritores: Etnobotânica; Plantas Medicinais; Odontologia.

\section{Abstract}

Ethnobotanical studies enable the rescue and preservation of popular knowledge of communities, in addition to which this information can be used in research for the production of herbal medicines in Brazil, which is still incipient, despite its enormous plant biodiversity. We aim to carry out an ethnobotanical study of medicinal plants indicated and used by the Araruna population. Two different structured questionnaires were used to obtain socioeconomic and ethnobotanical data, one for the raizeiros and the other for the users of the Unified Health System (SUS). Most of the interviewees (66.5\%) use medicinal plants. Of these, $52.63 \%$ live in the rural area and $47.37 \%$ live in the urban area. In addition, $27.5 \%$ obtained the plants at home and $27 \%$ at other places, such as at relatives' and friends' houses or on the neighbors' site. Regarding how the knowledge about the use of medicinal plants was acquired, $61 \%$ reported having obtained with the family. The plants for dental purposes most sold by raizeiros were: boldo, camomila, canela, cajueiroroxo, alecrim, alho, babatenon, aroeira, sabugueira, cravo, romã e ervadoce. The plants most indicated and used by the users of the Unified Health System (SUS) were: erva-cidreira, capim santo, boldo, sabugueira, hortelã da folha miúda, malva rosa, romã, hortelã da folha grossa, eucalipto, erva-doce, mastruz, louro, cajueiro roxo, alecrim, arruda e anador. The population of Araruna makes use of medicinal plants for the treatment of several health problems, with knowledge acquired in a popular way and without professional guidance on posology and / or possible toxicity of these plants.

Descriptors: Ethnobotany; Plants, Medicinal; Dentistry.

\section{Resumen}

Los estudios etnobotánicos permiten el rescate y la preservación del conocimiento popular de las comunidades, además de que esta información se puede utilizar en la investigación para la producción de hierbas medicinales en Brasil, que aún es incipiente, a pesar de su enorme biodiversidad vegetal. Nuestro objetivo es realizar un estudio etnobotánico de plantas medicinales indicadas y utilizadas por la población de Araruna. Se utilizaron dos cuestionarios estructurados diferentes para obtener datos socioeconómicos y etnobotánicos, uno para el "raeiro" y otro para los usuarios del Sistema Único de Salud (SUS). La mayoría de los encuestados (66.5\%) usan plantas medicinales. De estos, el 52,63\% vive en zonas rurales y el $47,37 \%$ en zonas urbanas. Además, el $27.5 \%$ obtuvo las plantas en el hogar y el $27 \%$ en otros lugares, como en el hogar de familiares y amigos o en el sitio de los vecinos. En cuanto a cómo se adquirió el conocimiento sobre el uso de plantas medicinales, el $61 \%$ dijo que lo había obtenido con la familia. Las plantas con fines dentales más vendidas por los "raçoeiros" fueron: boldo, manzanilla, canela, anacardo, romero, ajo, babatenon, aroeira, saúco, clavo, granada e hinojo. Las plantas más indicadas y utilizadas por los usuarios del Sistema Único de Salud (SUS) fueron: bálsamo de limón, capim santo, arándano, saúco, menta de hoja pequeña, malva rosa, granada, menta de hoja gruesa, eucalipto, hinojo, mastruz, laurel, anacardo morado, romero, rue y anador. La población de Araruna utiliza plantas medicinales para el tratamiento de diversos problemas de salud, con conocimientos adquiridos de manera popular y sin orientación profesional sobre la dosificación y / o posible toxicidad de estas plantas.

Descriptores: Etnobotánica; Plantas Medicinales; Odontología.

INTRODUÇÃO

O uso de plantas medicinais é um reflexo da realidade de parte da população brasileira, que possui um acesso limitado aos programas de saúde pública. Dessa forma, fazem uso do conhecimento etnobotânico para o tratamento das condições que afetam a saúde, por meio do 
uso da fitoterapia ${ }^{1}$. A fitoterapia é um campo das ciências da saúde que utiliza plantas para o tratamento de doenças e/ou modulador de saúde e funções biológicas ${ }^{2}$.

Sendo assim, o Brasil, que detém a maior diversidade biológica do mundo, contando com uma rica flora, desperta interesses de comunidades científicas internacionais para 0 estudo, conservação e utilização racional destes recursos, 0 que pode contribuir para 0 desenvolvimento de novos medicamentos produzidos a partir de plantas ${ }^{3,4}$.

Devido a essa grande diversidade das espécies vegetais, o Brasil destaca-se pela larga utilização de plantas para fins medicinais. Portanto, estudar o conhecimento populacional a respeito do mundo vegetal provê informações primárias como indicações e os modos de utilização das plantas medicinais ${ }^{5}$. Essas informações podem ser usadas como indicadores para 0 aprofundamento de pesquisas com relação às atividades biológicas, mecanismo de ação e toxicidade das plantas.

Estas plantas, quando indicadas para 0 tratamento das doenças bucais, apresentam uma alternativa de tratamento, visto que a maioria da população no Brasil não tem acesso à compra de produtos farmacêuticos sintéticos ${ }^{6}$. Porém, o reconhecimento oficial da fitoterapia na odontologia do Brasil veio acompanhado de diversas lacunas na pesquisa científica sobre plantas medicinais, como deficiências de informações sobre posologias, método de preparo e toxicidade desses produtos naturais ${ }^{5}$. $\mathrm{Na}$ prática clínica, ainda há uma grande necessidade de capacitação dos profissionais de saúde com relação à prescrição de fitoterápicos e drogas vegetais ${ }^{7}$ e, dessa forma, a população tendem a fazer o uso das plantas por conhecimento passado pelos seus ancestrais.

Nessa perspectiva, nosso objetivo foi realizar um levantamento etnobotânico para avaliar se a população do município de Araruna, PB, faz uso de plantas medicinais para o tratamento de doenças bucais e/ou sistêmicas, bem como identificar as indicações terapêuticas, modo de preparos e forma de utilização das plantas indicadas.

MATERIAL E MÉTODO

- Caracterização e área do estudo

Esta pesquisa foi realizada no período de março a junho de 2011, na cidade de Araruna ( $06^{\circ} 33^{\prime} 30^{\prime \prime} \mathrm{s}$; $35^{\circ} 44^{\prime} 30^{\prime \prime} \mathrm{W}, 570 \mathrm{~m}$ alt.), localizada na região do Curimataú Oriental da Paraíba, Nordeste do Brasil. Trata-se de um estudo do tipo transversal, cuja população foi representada pelos raizeiros atuantes na feira livre do município de Araruna e pelos usuários do SUS nas suas residências, nas zonas rural e urbana da cidade.

- Coleta dos dados

A população de estudo foi os raizeiros da feira livre municipal da cidade Araruna, PB e com os usuários do Sistema Único de Saúde (SUS). A coleta de dados foi feita com todos os raizeiros presentes durante a feira livre. Para os usuários do SUS foi realizado um sorteio pelo método de amostragem simples sem reposição das Unidades Básicas de Saúde (UBS). Foram sorteadas duas UBS dentre as oito existentes no município, sendo uma da zona urbana e outra da zona rural, além de um Agente Comunitário de Saúde (ACS) de cada uma das unidades. De cada microárea atendida pelo ACS, foram sorteadas cinquenta casas, totalizando duzentas casas, sendo o questionário aplicado para um residente de cada casa. Os dados foram coletados por meio de dois questionários estruturados diferenciados, um para os raizeiros e outro para os usuários do SUS. Em uma primeira etapa, foram entrevistados todos os raizeiros atuantes na feira livre da cidade. Em uma segunda etapa, os moradores da zona rural e urbana de Araruna foram entrevistados. O questionário foi utilizado para coleta de dados socioeconômicos e informações sobre as plantas medicinais, como a forma que são utilizadas suas indicações.

- Análise estatística

Os dados obtidos neste estudo foram tabulados em programa SPSS (Statiscal Package for Social Science) versão 20.0. A análise foi descritiva, com apresentação de dados em percentuais de frequência.

- Aspectos éticos

Essa pesquisa foi aprovada no CEP/UEPB com parecer no 0759.0.133.000-11, respeitando a resolução 466/12 do CNS.

RESULTADOS

A média de idade entre dos raizeiros entrevistados foi de 46 anos, sendo a maioria do sexo masculino. Oitenta por cento deles adquiriram o conhecimento sobre as plantas por meio de familiares e $20 \%$ por meio do comércio na própria feira. Com relação a escolaridade, $60 \%$ dos entrevistados não concluíram o ensino fundamental e $40 \%$ deles eram analfabetos.

Todos os raizeiros relataram vender plantas medicinais para fins terapêuticos à população da cidade e todas as plantas vendidas na feira de Araruna foram provenientes da própria cidade e de cidades 
que fazem parte da microrregião do Curimataú Oriental da Paraíba. As plantas para fins odontológicos mais vendidas pelos raizeiros foram: boldo, camomila, canela, cajueiro roxo, alecrim, alho, babatenon, aroeira, sabugueiro, cravo, romã e erva-doce.

Com relação à população usuária do SUS, como não houve perda da amostra, todos os 200 usuários foram entrevistados. A maioria mulher, parda e com idade entre os 30 e 59 anos. Setenta e seis por cento eram naturais da cidade de Araruna-PB, e com ensino fundamental incompleto (Tabela 1).

Tabela 1 - Distribuição da amostra de usuários do Sistema Único de Saúde de Araruna (PB) - Brasil segundo variáveis sociodemográficas

\begin{tabular}{|c|c|c|}
\hline Variáveis & $\mathbf{N}$ & $\%$ \\
\hline \multicolumn{3}{|l|}{ Sexo } \\
\hline Masculino & 28 & 14,0 \\
\hline Feminino & 172 & 86,0 \\
\hline \multicolumn{3}{|l|}{ Idade } \\
\hline Até 29 anos & 36 & 18,0 \\
\hline 30 a 59 anos & 121 & 60,5 \\
\hline 60 ou mais & 42 & 21,0 \\
\hline Não Informado & 1 & 0,5 \\
\hline \multicolumn{3}{|l|}{ Naturalidade } \\
\hline Araruna & 152 & 76,0 \\
\hline Outra Cidade & 48 & 24,0 \\
\hline \multicolumn{3}{|l|}{ Escolaridade } \\
\hline Não estudou & 39 & 19,5 \\
\hline Ensino Fundamental Incompleto & 81 & 40,5 \\
\hline Ensino Fundamental Completo & 28 & 14,0 \\
\hline Ensino Médio Incompleto & 4 & 2,0 \\
\hline Ensino Médio Completo & 35 & 17,5 \\
\hline Ensino Superior Incompleto & 5 & 2,5 \\
\hline Ensino Superior Completo & 8 & 4,0 \\
\hline \multicolumn{3}{|l|}{ Etnia } \\
\hline Branco & 28 & 14,0 \\
\hline Preto & 41 & 20,5 \\
\hline Pardo & 130 & 65,0 \\
\hline Amarelo & 1 & 0,5 \\
\hline Total & 200 & 100 \\
\hline
\end{tabular}

As informações relativas à utilização das plantas pela população, assim como a parte utilizada, a forma e a finalidade do uso, para cada planta, estão descritas nas tabelas 2 e 3.

Tabela 2: Informações gerais sobre as plantas utilizadas pela população de Araruna (PB) - Brasil

\begin{tabular}{|c|c|c|}
\hline Variáveis & $\mathbf{N}$ & $\%$ \\
\hline \multicolumn{3}{|l|}{ O senhor (a) faz uso de alguma planta medicinal? } \\
\hline Sim & 133 & 66,5 \\
\hline Não & 67 & 33,5 \\
\hline \multicolumn{3}{|l|}{ Local de obtenção das plantas (local de plantio) } \\
\hline Sítio particular & 23 & 11,5 \\
\hline Domicílio & 55 & 27,5 \\
\hline Outro & 54 & 27,0 \\
\hline Não informado & 68 & 34,0 \\
\hline \multicolumn{3}{|c|}{ Como foi adquirido o conhecimento sobre o uso de plantas medicinais } \\
\hline Família & 122 & 61,0 \\
\hline Amigos & 9 & 4,5 \\
\hline Livros & 1 & 0,5 \\
\hline Não informado & 68 & 34,0 \\
\hline \multicolumn{3}{|l|}{ Este conhecimento é transmitido } \\
\hline Sim & 122 & 61,0 \\
\hline Não & 9 & 4,5 \\
\hline Não Informado & 69 & 34,5 \\
\hline \multicolumn{3}{|l|}{ A quem este conhecimento é transmitido } \\
\hline Familiares & 93 & 46,5 \\
\hline Amigos & 29 & 14,5 \\
\hline Não Informado & 78 & 39,0 \\
\hline \multicolumn{3}{|l|}{ Forma de transmissão do conhecimento } \\
\hline Explanação Oral & 90 & 45,0 \\
\hline Demonstração prática da utilização da planta & 32 & 16,0 \\
\hline Não Informado & 78 & 39,0 \\
\hline \multicolumn{3}{|c|}{ Alguma Planta utilizada já apresentou algum efeito colateral } \\
\hline Sim & 1 & 0,5 \\
\hline Não & 148 & 74,0 \\
\hline Não informado & 51 & 25,5 \\
\hline Total & 200 & 100 \\
\hline
\end{tabular}

Tabela 3: Informações sobre a parte utilizada, a forma e a finalidade do uso das plantas utilizadas pela população de Araruna (PB) - Brasil

\begin{tabular}{|c|c|c|c|c|c|c|c|}
\hline Plantas & $\begin{array}{l}\text { Faz } \\
\text { Uso }\end{array}$ & $\begin{array}{l}\text { Parte } \\
\text { Usada }\end{array}$ & $\mathbf{N}$ & $\begin{array}{l}\text { Forma de } \\
\text { uso }\end{array}$ & $\mathbf{N}$ & Finalidade do $u$ & \\
\hline \multirow{4}{*}{$\begin{array}{l}\text { Erva-Cidreira } \\
\text { (Melissa } \\
\text { officinalis) }\end{array}$} & 73 & Folha & 73 & $\begin{array}{l}\text { Chá (bebe } \\
\text { infusão) }\end{array}$ & 72 & Dor de cabeça & 1 \\
\hline & & & & Outros & 1 & Tosse & 2 \\
\hline & & & & & & Outro & 69 \\
\hline & & & & & & Não sabe indicar & 1 \\
\hline \multirow{2}{*}{$\begin{array}{l}\text { Capim Santo } \\
\text { (Cymbopogon } \\
\text { citratus) }\end{array}$} & 71 & Folha & 71 & $\begin{array}{l}\text { Chá (bebe } \\
\text { infusão) }\end{array}$ & 71 & Dor de cabeça & 1 \\
\hline & & & & & & Outro & 70 \\
\hline $\begin{array}{l}\text { Boldo } \\
\text { (Peumus boldus) }\end{array}$ & 40 & Folha & 40 & $\begin{array}{l}\text { Chá (bebe } \\
\text { infusão) }\end{array}$ & 40 & Outro & 40 \\
\hline \multirow{3}{*}{$\begin{array}{l}\text { Sabugueiro } \\
\text { (Sambucus nigra) }\end{array}$} & 32 & Folha & 9 & $\begin{array}{l}\text { Chá (bebe } \\
\text { infusão) }\end{array}$ & 32 & Dor de cabeça & 2 \\
\hline & & Flores & 23 & & & Tosse & 6 \\
\hline & & & & & & Outro & 24 \\
\hline \multirow{2}{*}{$\begin{array}{l}\text { Hortelã } \mathbf{d a} \\
\text { Folha Miúda } \\
\text { (Mentha villosa) }\end{array}$} & 31 & Folha & 31 & $\begin{array}{l}\text { Chá (bebe } \\
\text { infusão) }\end{array}$ & 25 & Tosse & 4 \\
\hline & & & & Outros & 6 & Outro & 27 \\
\hline \multirow{3}{*}{$\begin{array}{l}\text { Malva Rosa } \\
\text { (Alcea rósea) }\end{array}$} & 30 & Folha & 30 & $\begin{array}{l}\text { Chá (bebe } \\
\text { infusão) }\end{array}$ & 29 & Tosse & 6 \\
\hline & & & & Outros & 1 & Outro & 23 \\
\hline & & & & & & Não sabe indicar & 1 \\
\hline \multirow{4}{*}{$\begin{array}{l}\text { Romã } \\
\text { (punica } \\
\text { granatum) }\end{array}$} & 23 & Caule & 1 & $\begin{array}{l}\text { Chá (bebe } \\
\text { infusão) }\end{array}$ & 12 & Dor de garganta & 2 \\
\hline & & Casca & 17 & $\begin{array}{l}\text { Tópico } \\
\end{array}$ & 1 & Inflamação & 20 \\
\hline & & Fruto & 5 & Gargarejo & 6 & Outro & 1 \\
\hline & & & & Outros & 4 & & \\
\hline \multirow{3}{*}{$\begin{array}{l}\text { Hortelã da } \\
\text { Folha Grossa } \\
\text { (Plectranthus } \\
\text { amboinicus } \\
\text { (Lour.) Spreng) }\end{array}$} & 21 & Folha & 21 & $\begin{array}{l}\text { Chá (bebe } \\
\text { infusão) }\end{array}$ & 15 & Tosse & 12 \\
\hline & & & & Outros & 6 & Outro & 8 \\
\hline & & & & & & Não sabe indicar & 1 \\
\hline \multirow[t]{2}{*}{$\begin{array}{l}\text { Eucalipto } \\
\text { (Eucalyptus) }\end{array}$} & 20 & Folha & 20 & $\begin{array}{l}\text { Chá (bebe } \\
\text { infusão) }\end{array}$ & 20 & Tosse & 2 \\
\hline & & & & & & Outro & 18 \\
\hline \multirow{2}{*}{$\begin{array}{l}\text { Erva- Doce } \\
\text { (Carum carvi) }\end{array}$} & 17 & Folha & 17 & $\begin{array}{l}\text { Chá (bebe } \\
\text { infusão) }\end{array}$ & 17 & Tosse & 1 \\
\hline & & & & & & Outro & 16 \\
\hline \multirow{3}{*}{$\begin{array}{l}\text { Mastruz } \\
\text { (Chenopodium } \\
\text { ambrosioides) }\end{array}$} & 12 & Folha & 12 & $\begin{array}{l}\text { Chá (bebe } \\
\text { infusão) }\end{array}$ & 8 & Tosse & 5 \\
\hline & & & & Outros & 4 & Inflamação & 1 \\
\hline & & & & & & Outro & 6 \\
\hline $\begin{array}{l}\text { Louro } \\
\text { (Laurus nobilis) }\end{array}$ & 12 & Folha & 12 & $\begin{array}{l}\text { Chá (bebe } \\
\text { infusão) }\end{array}$ & 12 & Outro & 12 \\
\hline \multirow{3}{*}{$\begin{array}{l}\text { Cajueiro Roxo } \\
\text { (Anacardium } \\
\text { occidentale) }\end{array}$} & 12 & Caule & 4 & $\begin{array}{l}\text { Chá (bebe } \\
\text { infusão) }\end{array}$ & 7 & Inflamação & 12 \\
\hline & & Casca & 8 & Tópico & 1 & & \\
\hline & & & & Outros & 4 & & \\
\hline $\begin{array}{l}\text { Alecrim } \\
\text { (Rosmarinus } \\
\text { officinalis) }\end{array}$ & 11 & Folha & 11 & $\begin{array}{l}\text { Chá (bebe } \\
\text { infusão) }\end{array}$ & 11 & Outro & 11 \\
\hline \multirow{3}{*}{$\begin{array}{l}\text { Arruda } \\
\text { (Ruta } \\
\text { graveolens) }\end{array}$} & 9 & Folha & 9 & $\begin{array}{l}\text { Chá (bebe } \\
\text { infusão) }\end{array}$ & 7 & Dor de cabeça & 3 \\
\hline & & & & Tópico & 2 & Inflamação & 1 \\
\hline & & & & & & Outro & 5 \\
\hline \multirow{3}{*}{$\begin{array}{l}\text { Anador } \\
\text { (Justicia } \\
\text { pectoralis) }\end{array}$} & 9 & Folha & 9 & $\begin{array}{l}\text { Chá (bebe } \\
\text { infusão) }\end{array}$ & 9 & Dor de dente & 1 \\
\hline & & & & & & Dor de cabeça & 6 \\
\hline & & & & & & $\begin{array}{l}\text { Outro } \\
\end{array}$ & 2 \\
\hline
\end{tabular}

\section{DISCUSSÃO}

Apesar de muitas plantas da região nordeste serem utilizadas para fins terapêuticos odontológicos, existem poucos estudos científicos que comprovem suas atividades biológicas $^{8,9}$. No Brasil, considerando a ampla diversidade de espécies vegetais, bem como a riqueza étnico-cultural, o uso popular de plantas medicinais é muito relevante. Por isso, os estudos etnobotânicos são fundamentais, uma vez que possibilitam o resgate e a preservação dos conhecimentos populares das comunidades envolvidas $^{10}$.

Em nosso estudo, a maioria dos entrevistados foi do sexo feminino, fato observado em estudos semelhantes realizados em 2009, como o de Jesus et al. ${ }^{11}$ e Santos et al. $^{12}$. Estes resultados podem estar relacionados ao fato da entrevista ser realizada em horário comercial e, em geral, nos municípios do interior os homens estão no trabalho e as mulheres 
envolvidas com as tarefas domésticas. Ceolin et al. $2011^{13}$ evidenciaram a importância das mulheres na transmissão do conhecimento entre as gerações e a responsabilidade pela execução do cuidado em saúde na família, utilizando-se das plantas medicinais para a sua realização.

Com relação à transmissão do conhecimento popular, as informações sobre os hábitos e cuidados com a saúde, como o uso de plantas medicinais, se propagam oralmente entre os membros da família ${ }^{14}$. Esta informação foi confirmada pelos nossos achados e corrobora os resultados de Alves \& Silva ${ }^{15}$ e Veiga Júnior ${ }^{16}$. Ainda cabe destacar que a família é um sistema no qual se conjugam valores, crenças, conhecimentos e práticas, formando um modelo explicativo de saúde/doença, por meio do qual a família desenvolve sua dinâmica de funcionamento, promovendo a saúde, prevenindo e tratando a doença de seus membros ${ }^{14}$.

Uma preocupação é a possível toxicidade, contraindicações, interações medicamentosas com medicamentos alopáticos e/ou efeitos adversos das plantas medicinais ${ }^{6}$. Em nosso estudo identificamos que a transmissão do conhecimento a respeito do uso das plantas medicinais é feito majoritariamente de forma popular. Desse modo, torna-se importante a participação dos profissionais na orientação da população no âmbito da atenção básica em saúde.

Tem sido relatado que a maioria dos profissionais de saúde não tem conhecimento ou se sentem inseguros para orientar a população sobre a utilização de plantas medicinais ${ }^{17}$. Foi observada uma grande necessidade de capacitação e motivação desses profissionais para a utilização correta e segura das plantas medicinais e fitoterapia ${ }^{7}$.

Foi verificado anteriormente que informantes mais idosos são os que possuem maior informação sobre o uso de plantas medicinais $^{18}$. Para Veiga Júnior ${ }^{16}$, o conhecimento das indicações terapêuticas das plantas medicinais geralmente é uma atribuição das pessoas idosas, que também são responsáveis pelo preparo das formulações à base de plantas. Porém, observamos em nosso estudo que as informações obtidas referentes ao uso das plantas foram obtidas de pessoas mais jovens. Entretanto, este dado está relacionado com a média de idade da nossa amostra e não com o fato de os entrevistados mais jovens possuírem maior conhecimento, uma vez que as informações foram obtidas por meio de seus ancestrais.
Quanto à forma de preparo das plantas, a maioria delas são preparadas em forma de chá, seja por decocto ou infuso, porque são maneiras mais fáceis e rápidas. $O$ que coincide com o levantamento feito por Borba e Macêdo ${ }^{5}$, no qual a forma mais usada no preparo do medicamento caseiro foi o chá por decocto, seguido pelo chá por infusão, observando-se que uma espécie vegetal pode ter mais de uma maneira de preparo para sua utilização terapêutica. O mesmo estudo mostrou que a parte mais utilizada pelos usuários era a folha, fato confirmado por nossos achados.

A maioria dos usuários de plantas medicinais do nosso estudo as adquire em canteiros de sua própria casa, sítio de amigos ou na feira livre da cidade, corroborando o achado de Veiga Júnior ${ }^{16}$. No entanto, o mesmo estudo demonstrou que uma parte dos entrevistados se preocupa com a origem/qualidade do farmacógeno utilizado, adquirindo-o em lojas específicas de produtos naturais e/ou em farmácias de manipulação. É importante enfatizar que a amostra do estudo, acima referido, foi composta por profissionais de saúde, além da população, explicando o maior cuidado com a obtenção das plantas.

Em síntese, o uso de plantas medicinais para o tratamento de enfermidade é uma prática antiga na história da humanidade e a Organização Mundial de Saúde (OMS) traz recomendações quanto à difusão dos conhecimentos necessários ao uso das plantas medicinais, pois, embora sejam popularmente consideradas inócuas, frequentemente possuem propriedades tóxicas desconhecidas pela população, o que acarreta risco à saúde dos seus usuários. Entretanto, a utilização da medicina informal ou popular realizada por indivíduos que se valiam de seus diferentes saberes é bastante difundida no Brasil onde algumas plantas consideradas medicinais são comercializadas de forma não controlada. Isso pode ser ocasionado devido ao alto custo dos medicamentos industrializados e pelo difícil acesso à assistência médica, entre outros motivos $^{19}$. No entanto, em quase totalidade de nossa amostra, não foi relatado efeito colateral devido ao uso dos espécimes vegetais.

CONCLUSÃO

As plantas de uso odontológico mais vendidas pelos raizeiros foram: boldo, camomila, canela, cajueiro roxo, alecrim, alho, babatenon, aroeira, sabugueira, cravo, romã e erva-doce. Já as plantas mais utilizadas pela população da zona urbana e da zona rural de Araruna (PB) - Brasil foram: erva-cidreira, capim 
santo, boldo, sabugueira, hortelã da folha miúda, malva rosa, romã, hortelã da folha grossa, erva-doce, eucalipto, mastruz, louro, cajueiro roxo, alecrim, arruda e anador. A população do município de Araruna (PB) - Brasil faz uso de plantas medicinais para o tratamento de doenças bucais e/ou sistêmicas, sendo as indicações terapêuticas mais comuns para o tratamento da dor, tosse e inflamação. A forma de preparo mais comum para utilização das plantas é na forma de chá e decocto. O conhecimento é adquirido de forma popular e sem orientação profissional sobre posologia e/ou possível toxicidade dessas plantas, o que indica a importância do investimento na imersão dos profissionais de saúde (médicos, enfermeiros, dentistas e farmacêuticos) no conhecimento de fitoterapia para orientação da população.

\section{REFERÊNCIAS}

1. Santos MRA Lima MR, Ferreira MGR. Uso de plantas medicinais pela população de Ariquemes, em Rondônia. Hortic bras. 2008; 26(2):244-50.

2. Falzon CC, Balabanova A. Phytotherapy: An Introduction to Herbal Medicine. Prim Care. 2017;44:217-27.

3. Fontes DJ, Coelho VAT, Gomes, FT. Uso de plantas medicinais pelos moradores da comunidade de Conceição de Ibitipoca, MG. Rev Bras Biociênc. Porto Alegre. 2007;5(1): 237-39.

4. Souza CD, Felfili JM. Uso de Plantas Medicinais na Região do Alto Paraíso de Goiás, GO, Brasil. Acta Bot Bras. 2006;20(1):135-42.

5. Borba AM, Macedo, M. Plantas medicinais usadas para a saúde bucal pela comunidade do bairro Santa Cruz, Chapada dos Guimarães, MT, Brasil. Acta Bot Bras. 2006;20(4):771-82.

6. França WFA, Marques MMMR, Lira KDL, Higino M. E. Terapêutica com plantas medicinais nas doenças bucais: a percepção dos profissionais no programa de saúde da família do Recife. Odontol clín cient. 2007;6(3): 223-37.

7. Nascimento Júnior BJ1, Tínel LO, Silva ES, Rodrigues, LA, Freita TON, Nunes XP, Amorim ELC. Avaliação do conhecimento e percepção dos profissionais da estratégia de saúde da família sobre o uso de plantas medicinais e fitoterapia em Petrolina-PE, Brasil. Rev bras plantas med. 2016;18:57-66.

8. Agra MF, Freitas PF, Barbosa Filho JM. Synopsis of the plants known as medicinal and poisonous in Northeast of Brazil. Rev bras farmacogn. 2007;17:114-140.

9. Agra MF, Silva KN, Basílio IJLD, França, PF, Barbosa Filho JM. Survey of medicinal plants used in the region Northeast of Brazil. Rev bras farmacogn. 2008;18(3):472-508.

10. Garlet TMB, Irgang BE. Plantas medicinais utilizadas na medicina popular por mulheres trabalhadoras rurais de Cruz Alta, Rio Grande do Sul, Brasil. Rev bras plantas med. 2001;4: 9-18.

11. Jesus NZT, Lima JCS, Silva RM, Espinosa MM, Martins DTO. Levantamento etnobotânico de plantas popularmente utilizadas como antiúlceras e anti-inflamatórias pela comunidade de Pirizal, Nossa Senhora do Livramento - MT, Brasil. Rev bras farmacogn. 2009;19(1):130-39.

12. Santos EB, Dantas GS, Santos HB, Diniz MFFM, Sampaio FC. Estudo etnobotânico de plantas medicinais para problemas bucais no município de João Pessoa, Brasil. Rev bras farmacogn. 2009;19(1):321-24.

13. Ceolin T, Heck RM, Barbieri RL, Schwartz E, Muniz RM, Pillon CN. Plantas medicinais: transmissão do conhecimento nas famílias de agricultores de base ecológica no Sul do RS. Rev esc enferm USP. 2011;45(1):47-54.

14. Elsen, I. Cuidado familial: uma proposta inicial de sistematização. 2. ed. In: Elsen I, Marcon SS, Silva MRS., organizadores. O viver em família e sua interface com a saúde e a doença. Maringá: EDUEM; 2004. p.19-28.

15. Alves DL, Silva CR. Fitohormônios: abordagem natural da terapia hormonal. São Paulo: Atheneu, 2002.

16. Veiga Junior, FV. Estudo do consumo de plantas medicinais na Região Centro-Norte do Estado do Rio de Janeiro: aceitação pelos profissionais de saúde e modo de uso pela população. Rev bras farmacogn. 2008;18(2): 308-13.

17. Lopes MA, Obici S, Albiero AL. Conhecimento e intenção de uso da fitoterapia em uma unidade básica de saúde. ICSA.2012;1(1):53-9.

18. Amaral CN, Guarim Neto G. Os quintais como espaços de conservação e cultivo de alimentos: um estudo na cidade de Rosário Oeste (Mato Grosso, Brasil). Bol Mus Para Emílio Goeldi Ciências Humanas, Belém, 2008;3(3):329-41.

19. Bochner R, Fiszon JT, Assis MA, Avelar KES. Problemas associados ao uso de plantas medicinais comercializadas no Mercadão de Madureira, município do Rio de Janeiro, Brasil. Rev bras plantas med. 2012;14(3):537-47. 


\section{CONFLITO DE INTERESSES}

Os autores declaram não haver conflitos de interesse

\section{AUTOR PARA CORRESPONDENCIA}

Andréa Cristina Barbosa da Silva

Departamento de Farmácia, Centro de ciências

Biológicas. Universidade Estadual da Paraíba - UEPB

Campus I. R. Baraúnas, 351, Bairro Universitário,

58429-500 Campina Grande-PB, Brasil.

Tel: (83) 3315-3300.

E-mail: andreacbsilva@gmail.com

Submetido em 01/07/2020

Aceito em 23/10/2020 